\title{
НЕЧЕТНАЯ СИММЕТРИЯ ВЕКТОРА ВЕСОВЫХ КОЭФФИЦИЕНТОВ СИММЕТРИЧНЫХ АНТЕННЫХ РЕШЕТОК С ЛИНЕЙНЫМИ ОГРАНИЧЕНИЯМИ
}

\author{
в. И. ДЖИГАН
}

\author{
Национальный исследовательский университет \\ «Московский институт электронной техники», \\ Россия, Москва, 124498, Зеленоград, пл. Шокина, 1
}

\begin{abstract}
Аннотация. В работе приведено доказательство нечетной симметрии вектора весовых коэффициентов, полученных на основе критерия наименьших квадратов, в симметричной линейной адаптивной антенной решетке с линейными ограничениями и требуемым сигналом. Пары симметричных элементов такого вектора являются комплексно-сопряженными по отношению друг к другу. Для обеспечения данного свойства вектор ограничиваемых параметров (значения диаграммы направленности антенной решетки в интересуемых направлениях) должен быть действительным, но не обязательно симметричным. Нечетная симметрия векторов входных сигналов и весовых коэффициентов антенной решетки позволяет разрабатывать для такой решетки адаптивные алгоритмы в арифметике действительных чисел. В этом случае число арифметических операций таких алгоритмов, приходящихся на одну итерацию, примерно в два или четыре раза меньше эквивалентного числа действительных арифметических операций аналогичных алгоритмов в арифметике комплексных чисел. В работе представлены результаты сравнительного моделирования алгоритмов в арифметике комплексных и действительных чисел. Они показывают, что адаптивный алгоритм, использующий арифметику действительных чисел, обеспечивает в 1,5-2 раза более короткий переходный процесс и более глубокие провалы (2-3 дБ) в установившемся режиме в диаграмме направленности антенной решетки в направлениях на источники адаптивно подавляемых помех, чем алгоритм в арифметике комплексных чисел.
\end{abstract}

Ключевые слова: адаптивная антенная решетка; алгоритм адаптивной фильтрации; нечетная симметрия; RLS

\section{ВВЕДЕНИЕ}

Адаптивная обработка сигналов - одна из ключевых технологий, используемых в оборудовании современных систем связи, радиолокации, радионавигации, а также бытовой техники. Одним из элементов такого оборудования часто является направленная антенна, которая при реализации в виде антенной решетки позволяет немеханическим способом перемещать основной лепесток диаграммы направленности (ДН), повышать отношение сигнал-тепловой шум в выходном сигнале решет- ки, а также уменьшать в этом сигнале уровень помех от внешних источников за счет изменения формы ДН.

Антенные решетки, обладающие последним свойством, называются адаптивными (ААР) [1]. В таких решетках подавление помех обеспечивается за счет образования провалов в ДН в неизвестных на приемной стороне направлениях на источники помех. Изменение формы ДН достигается за счет изменения значений весовых коэффициентов, с помощью которых сигналы в каждом из каналов ААР взве- 


\section{БИБЛИОГРАФИЧЕСКИЙ СПИСОК}

1.Compton, R.T. Adaptive Antennas: Concepts and Performance. Prentice Hall, 1988. 448 p.

2. Diniz, P. S. R. Adaptive Filtering. Algorithms and Practical Implementation, 4th ed. Springer, 2013. DOI: 10.1007/978-1-4614-4106-9.

3. Farhang-Boroujeny, B. Adaptive Filters: Theory and Applications, 2nd ed. John Willey and Sons, 2013. $778 \mathrm{p}$.

4. Haykin, S. O. Adaptive Filter Theory, 5th ed. Pearson Education, 2014. 889 p.

5. Джиган, В. И. Адаптивная фильтрачия сигналов: теория и алгоритмы. М.: Техносфера, $2013.528 \mathrm{c}$.

6. Frost, O. L. "An algorithm for linearly constrained adaptive array processing," Proc. IEEE, Vol. 60, No. 8, P. 926-935, 1972. DOI: 10.1109/PROC.1972.8817.

7. Resende, L. S.; Romano, J. M. T.; Bellanger, M. G. "A fast least-squares algorithm for linearly constrained adaptive filtering," IEEE Trans. Signal Processing, Vol. 44, No. 5, P. 1168-1174, 1996. DOI: $10.1109 / 78.502329$.

8. Apolinario, J. A.; Werner, S.; Diniz, P. S. R.; Laakso, T. I. "Constrained normalized adaptive filters for CDMA mobile communications," Proc. of 9th European Signal Processing Conf., 8-11 Sept. 1998, Rhodes, Greece. IEEE, 1998. URI: https://ieeexplore.ieee.org/ document/7089625/.

9. De Campos, M. R. L.; Apolinario J. A. "The constrained affine projection algorithm - development and convergence issues," Proc. of First Balkan Conf. on Signal Processing, Communications, Circuits, and Syste$m s$, Istanbul, May 2000.

10. Cantoni, A.; Butler, P. "Properties of the eigenvectors of persymmetric matrices with applications to communication theory," IEEE Trans. Commun., Vol. 24, No. 8, P. 804-809, 1976. DOI: 10.1109/TCOM. 1976.1093391.

11. Nitzberg, R. "Application of maximum likelihood estimation of persymmetric covariance matrices to adaptive processing," IEEE Trans. Aerospace Electronic Syst., Vol. AES-16, No. 1, P. 124-127, 1980. DOI: $10.1109 /$ TAES.1980.308887.

12. Huarng, K.-C.; Yen, C.-C. "A unitary transformation method for angle-of-arrival estimation,"
IEEE Trans. Signal Processing, Vol. 39, No. 4, P. 975-977, 1991. DOI: https://doi.org/10.1109/78.80927.

13. Зарицкий, В. И.; Кокин, В. Н.; Леховицкий, Д. И.; Саламатин, В. В. "Рекуррентные алгоритмы адаптивной обработки при центральной симметрии пространственно-временных каналов приема," Известия вузов. Радиофизика, Т. 28, № 7, С. 863-871, 1985.

14. Huarng, K.-C.; Teh, C.-C. "Adaptive beamforming with conjugate symmetric weights," IEEE Trans. Antennas Propag., Vol. 39, No. 7, P. 926-932, 1991. DOI: https://doi.org/10.1109/8.86911.

15. Zhang, L.; Liu, W.; Langley, R. J. "A class of constrained adaptive beamforming algorithms based on uniform linear arrays," IEEE Trans. Signal Processing, Vol. 58, No. 7, P. 3916-3922, 2010. DOI: 10.1109/TSP. 2010.2046078 .

16. Zhang, L.; Liu, W.; Langley, R. J. "A class of constant modulus algorithms for uniform linear arrays with a conjugate symmetric constraint," Signal Processing, Vol. 90, No. 9, P. 2760-2765, 2010. DOI: 10.1016/j. sigpro.2010.04.003

17. Zhang, L.; Liu, W.; Langley, R. J. "Adaptive beamforming with real-valued coefficients based on uniform linear arrays," IEEE Trans. Antennas Propag., Vol. 59, No. 3, P. 1047-1053, 2011. DOI: 10.1109/TAP. 2010.2103037 .

18. Zhang, L.; Liu, W.; Yu, L. "Performance analysis for finite sample MVDR beamformer with forward backward processing," IEEE Trans. Signal Processing, Vol. 59, No. 5, P. 2427-2431, 2011. DOI: 10.1109/TSP. 2011.2109957.

19. Xu, D.; He, R.; Shen, F. "Robust beamforming with magnitude response constraints and conjugate symmetric constraint," IEEE Commun. Lett., Vol. 17, No. 3, P. 561-564, 2013. DOI: 10.1109/LCOMM.2013.011 513.122688 .

20. Ратынский, М. В.; Петров, С. В. “Реализация алгоритмов обработки стохастических сигналов в действительной арифметике," Цифровая обработка сигналов, № 4, С. 22-24, 2013. URI: https://elibrary.ru/item. asp?id $=21197747$.

21. Liu, J.; Liu, W.; Liu, H.; Chen, B.; Xia, X.-G.; Dai, F. "Average SINR calculation of a persymmetric sample matrix inversion beamformer," IEEE Trans. Signal Processing, Vol. 64, No. 8, P. 2135-2145, 2016. DOI: $10.1109 /$ TSP.2015.2512527.

22. Lekhovytskiy, D. I. "To the theory of adaptive signal processing in systems with centrally symmetric receive channels," EURASIP J. Adv. Signal Process., Vol. 33, P. 1-11, 2016. DOI: 10.1186/s13634-016-0329-z.

23. Djigan, V. I. "Odd symmetry of weights vector in linearly-constrained adaptive arrays with desired signal," Proc. of Int. Conf. on Antennas Theory and Techniques, 24-27 May 2017, Kiev, Ukraine. IEEE, 2017, P. 140-144. DOI: $10.1109 /$ ICATT.2017.7972604.

24. Джиган, В. И. “Алгоритмы адаптивной линейно-ограниченной слепой обработки сигналов в цифровых антенных решетках с нечетной симметрией,", 
Цифровая обработка сигналов, № 2, С. 3-13, 2015. URI: https://elibrary.ru/item.asp?id=23833950.

25. Джиган, В. И. "Многолучевая адаптивная антенная решетка," Известия ЮФУ. Технические науки,
№ 2, C. 23-29, 2012. URI: http://izv-tn.tti.sfedu.ru/?p $=9410$. 